Dermatologische Zeitschrift. 1931;60:I-IV

\title{
Contents, Vol. 60, 1931
}

\section{Dermatologische Zeitsehrift}

Berichtsorgan der Berliner Dermatologíschen Gesellschaft Begründet von O. LASSAR Unter Mitwirkung von Sainz de Aja-Madrid; J. Almkvist-Stockholm; Ed. Arniлg-Hamburg; F. Bering - Essen; S. Bettmann-Heidelberg; Fr. Blumenthal-Berlin; H. Boas-Kopenhagen; C. Bruck-Altona; C. Bruhns-Berlin; E. Bruusgaard - Oslo; J. S. Covisa-Madrid; C. CronquistMalmö; J. Darier-Paris; E. Delbanco-Hamburg; K. Dohi-Tokio; U. Ebbecke-Bonn; W. FrieboesRostock; R. Früh-wald-Chemnitz; 0. Gans-Frankíurt; W.Gennerich-Kiel; C. Grouven-Halle; 0. Grütz-Elberîeld; R. Haberman $\pi$ - Hamburg ; L. Hauck-Erlangen; J. Heller-Berlin; W. HeuckMünchen; E. Hof-mann-Kassel; F. Jahnel-Mtinchen; A. Jesionek-Gießen; A. Jordan - Moskau; F. Julîusberg-Braunschweig; W. Kerl-Wien; W. Kolle - Frankfurt; P. Li nser-Tubingen; E. G. Graham Little-London; H. Löhe-Berlin; W. Lutz-Basel; S. Matsumoto-Kyoto; R. MatzenauerGraz; E. Meirowsky-Kòln; A. Memmesheimer-Tubingen; H. Meyer - Bremen; G. MiescherZurich; P. Mulzer-Hamburg; 0. Naegeli - Bern; E. Nathan-Nürnberg; G.Nobl-Wien; A. PasiniMai-land; F. Pinkas - Berlin; R. Polland - Qraz; C. Rasch-Kopenhagen; E. Riecke-Göttingen; H. Ritter-Hamburg; G. Rost-Freiburg; A. Ruete-Marburg; G. Scherber-Wien; W. I. SchmidtGießen; W. Scholtz - Königsberg; W. Schõnf eld - Greifswald; H. Th. Schreus - Düsseldorf; H. W. Siemens-Leiden; B. Spiethof f-Jena; C. Stern-Düsseldorf; R. Strempel-Bonn; A. Stühmer-

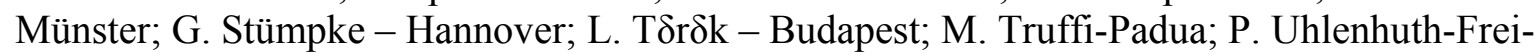
burg; H. Vörner - Leipzig; J. Werther-Dresden; Ch. J. White-Boston; K. Zieler - Würzburg ·t F. Zinsser-Köln; L. von Zumbusch-München; E. Zurhelle- Aachen und in Gemeinschaft mit

A. Alexander L. Arzt B. Bloch A. Buschke

Berlin Wien Zurich Berlin

K. Herxheimer V. Klingmüller

Frankfurt Kiel

herausgegeben von

Erich Hoffmann

Bonn

Band LX

BERLIN 1931

VERLAG VON S. KARGER

KARLSTRASSE 39

Mit zahlreichen Abbildungen im Text und einem Porträt

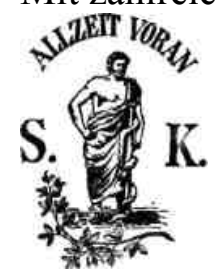


Alle Rechte vorbehalten

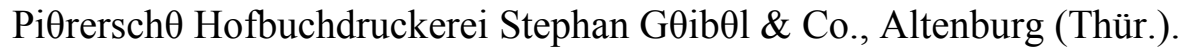

Inhaltsverzeichnis.

Originalarbeiten.

A $b$ ram son, L., Beitrag zu den Vergleichsuntersuchungen der Wa.E.

und der M. T. R 491

Beirach, J. S., Ein Inophoreseversuch mit unmittelbarer Einwirkung

auf Prostata und Samenbläschen in der Klinik der Gonorrhoe . . 175 Benedek, T., Über

Spalthefebefunde beim seborrhoischen Ekzem . . 381 Bernstein, P., Antwort auf die

Erwiderung von T. Benedek, Über

Spalthefebefunde beim seborrhoischen Ekzem $\quad 332$

Brauer, A., Über Heterochromíe der Haare 438

Cohn, Alfred, Verimpfung von Gonokokken auf Kaninchen 35

Curth, Wilhelm, Gemeinsames Vorkommen \ron multiplen Rumpf-

epitheliomen mit Parapsoriasis en plaques 256

Dittrich, 0., Über den direkten Tuberkelbazillennachweis beim Lupus

pernio und beim Sarkoid Boeck 395

Dörffel, J., Experimentelles und Klinisches über Krampfaderverödung.

Zweite Mitteilung 477

Fellner, M., Der Eínfiuß des Impfmalariaserums auf den Ablauf der

Luetinreaktion 41

Gitmul, L., siehe Müller, Hugo.

Grundmann, H., siehe Nathan, E.

Hämel, J., Die Behandlung des Trippers bei Frauen mit Pyrifer . . . 404

Hauck, Leo, Die Meinickesche Klämngsreaktion, eine sehr brauchbare

und leistungsfähige Untersuchungsmethode für die serologische

Syphílisdiagnose $\quad 379$

Hoffmann, Erich, Zur Frage der Syphilisimmunität, der Re- und Super-

infektion sowie der Reinduration 1

Lichen planus und Zisternenpunktion 165

Die praktische und wissenschaftliche Bedeutung der neuen Mikro-Schnellreaktion nach

Meinicke 221

Zur Frage der maximalen Frühbehandlung der primären und sekun-dären Syphilis 375

Hopf, Gustav, Über eine bisher nicht beschriebene disseminierte Kera-

tose (Akrokeratosis verruciformis) 227

Hörnicke, C. B., Künstliche Höhensonne und Vitaluxlampen .... 451 Isaak, Ludwig,

Abszeßbildung nach Varizenverödung mit Kalorose. 266

Kadisch, E., Die Hitzeresistenz einiger Hefen 48

Klövekorn, G. H., Zur Häufigkeit der Rektumsgonorrhoe 410

Leven, L., Zur Kenntnis der „Atrophodermia vermiculata” 250

Mallinckrodt-Haupt, Asta v., Lupus und Karzinom 138

Marquardt, F., siehe Schönfeld, W.

Mayr, Julius K., Die Reakíion des menschlichen Schweißes 413

Mühlpfordt, H., Rotlaufserumbehandlung oder Höhensonnenintensiv-

bestrahlung des Erysipeloids ? . .445

Müller, Hugo, und L. Gitmul, Goldbehandlung der Syphilis mit Sol- 
ganal B $\quad 153$

IV Inhaltsverzeichnis.

Seite

Nast, Otto, und Walter Riebe, Augenblickliches Urteil über die

Behandlung der weiblichen Gonorrhoe durch Impfmalaria 427

Nathan, E., und H. Grundmann, Über den Nachweis reaktionsver-stärkender Substanzen im Serum von Salvarsantoxidermien bei der Salvarsan-Hautreaktion salvarsanüberempfindlicher Personen .... 127

- und Fr. Stern, Über den Mineralgehalt der Haut unter normalen undpathologischenBedingungen. VI. Mitteilung: Über Schwankungen im Kalium-, Kalzium-, Chlor- und Wasscrgehalt der Kaninchen- und Meerschweinchenhaut bei gleíc·hmäßiger Ernährung und bei Hunger und Durst 299

Pachur, Reinhold, Quantitative Bestimmung des menschlichen Bluttalges 486

Riebe, Walter, siehe Nast, Otto.

Riebes, Ernst, Das R.G.B.G. und die Erfassung der Manner. . . . 455

Rock, Hans, Zur Arz¿reimittelbehandlung des Lupus vulgaris .... 421

Rohrbach, Richard, Über die Allgemeinbehandlung des Ekzems . . 14

Schönfeld, W., und F. Marquardt, Beiträge zu zwei Immunitäts-

reaktionen des Trippers 385

Schubert, Martin, Stürmische Provokation einer bislang erscheinungs-

freien Tabes durch Salvarsanbehandlung 169

Schumacher, Carl, Tierexperimenteller Beitrag zur Abhängigkeit des

Salvarsaneffektes vom Zustand des retikuloendothelialen Systems (R. S.) 469

Schwarzkopf, Arthur, Beitrag z $\mathrm{z}^{1 / 8} \mathrm{r}$ Atiologie der Trichotillomanie . 321

Serefis, S., Polymorphes Exanthem als Komplikation einer akzidentellen

Vakzinatíon 260

Silberstein, S., Zur Sicherung der Gonorrhoediagnose in der allgemeinen Praxis durch die Komplementbindungsreaktion und das

Kulturverfahren $\quad 464$

Stern, Fr., siehe Nathan, E.

Vohwinkel, K. H., Über das Vorkommen von apokrinen Driisen. . . 314

Gesellschaitsberichte.

Berliner Dermatologische Gesellschaft. Sitzung vom 8. VII. 1930 .... 51

- Sitzung vom 11. XI. 1930333

Sitzung der Dermatologischen Sektion am 10. IX. 1930 auf der Ver-sammlung Deutscher Naturforscher und Ärzte in Königsberg 1930, zugleich 18. Sitzung der Nordostdeutschen Dermatologischen Ver-

einigung $\quad 66$

Gemeinsame Tagung der Niederländischen Vereinigung von Dermatologen

und der Vereinigung rhemisch-westfälischer Dermatologen in Utrecht

am 31. V. und 1. VI. 1930189

Periodische Literatur. Zusammengestellt von Dr. Arthur Alexander-

Charlottenburg 71, 199, 268, 357

Buchbesprechungen $\quad 121$ 
Personalîen und Tagesnachrichten 126, 220, 297, 374

Zum Artikel „Editorial” von W. Allen Pusey 219

Sachregister 495

Natnenregister 503 